\title{
Rate-Distortion Optimization for Stereoscopic Video Streaming with Unequal Error Protection
}

\author{
A. Serdar Tan, ${ }^{1}$ Anil Aksay, ${ }^{2}$ Gozde Bozdagi Akar, ${ }^{2}$ and Erdal Arikan1 \\ ${ }^{1}$ Department of Electrical and Electronics Engineering, Bilkent University, 06800 Ankara, Turkey \\ ${ }^{2}$ Department of Electrical and Electronics Engineering, Middle East Technical University, 06531 Ankara, Turkey
}

Correspondence should be addressed to A. Serdar Tan, serdar@ee.bilkent.edu.tr

Received 1 October 2007; Revised 7 February 2008; Accepted 27 March 2008

Recommended by Aljoscha Smolic

\begin{abstract}
We consider an error-resilient stereoscopic streaming system that uses an H.264-based multiview video codec and a rateless Raptor code for recovery from packet losses. One aim of the present work is to suggest a heuristic methodology for modeling the end-toend rate-distortion (RD) characteristic of such a system. Another aim is to show how to make use of such a model to optimally select the parameters of the video codec and the Raptor code to minimize the overall distortion. Specifically, the proposed system models the RD curve of video encoder and performance of channel codec to jointly derive the optimal encoder bit rates and unequal error protection (UEP) rates specific to the layered stereoscopic video streaming. We define analytical RD curve modeling for each layer that includes the interdependency of these layers. A heuristic analytical model of the performance of Raptor codes is also defined. Furthermore, the distortion on the stereoscopic video quality caused by packet losses is estimated. Finally, analytical models and estimated single-packet loss distortions are used to minimize the end-to-end distortion and to obtain optimal encoder bit rates and UEP rates. The simulation results clearly demonstrate the significant quality gain against the nonoptimized schemes.
\end{abstract}

Copyright (C) 2009 A. Serdar Tan et al. This is an open access article distributed under the Creative Commons Attribution License, which permits unrestricted use, distribution, and reproduction in any medium, provided the original work is properly cited.

\section{Introduction}

The recent increase in interest for stereoscopic display systems and their growing deployment have spurred further research on efficient stereoscopic video streaming systems. Stereoscopic video is formed by the simultaneous capture of two video sequences corresponding to the left and right views of human visual system, which increases the amount of source data. Existing stereoscopic techniques compress the data by exploiting the dependency between the left and right views; however, the compressed video is more sensitive to data losses and needs added protection against transmission errors. To make matters more complicated, the rate of packet losses in the transmission channel is typically time varying. Hence, one faces a difficult joint source-channel coding problem, where the goal is to find the optimal balance between the distortion created by lossy source compression and the distortion caused by packet losses in the transmission channel. In this paper, we address this problem by (i) proposing a heuristic methodology for modeling the end-to- end RD characteristic of such a system, and (ii) dynamically adjusting the source compression ratio in response to channel conditions so as to minimize the overall distortion.

As opposed to stereoscopic video streaming, various studies exist in the literature for layered or nonlayered monoscopic video on optimal rate allocation and error resilient streaming on error prone channels such as packet erasure channel (PEC). The early studies on monoscopic video streaming mainly concentrate on nonlayered video and the optimal bit control and bit rate allocation for the video elements [1-4]. RD optimization is the most widely used optimization method for the quality of video, and it is a mechanism that aims to calculate optimal redundancy injection rate into the network, while adapting the video bit rate accordingly in order to match the available bandwidth estimate. Redundancy may be generated by means of either retransmissions or forward error correction (FEC) codes, and this redundancy is used to minimize the average distortion resulting from network losses during a streaming session [5-8]. Even though retransmission methods can be 


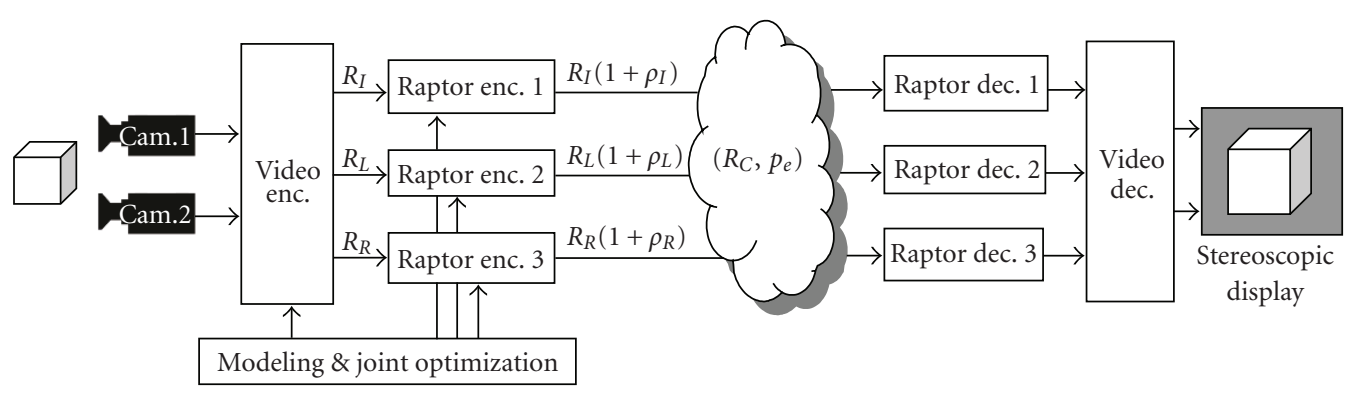

FIGURE 1: Overview of the stereoscopic streaming system.

used in video streaming applications as in [9], it may bring large latency for video display. On the other hand, FEC schemes insert protection before the transmission and do not utilize retransmissions. In literature, FEC methods are studied for video streaming as in [10-12].

A novel technique that recently becomes popular for error protection in lossy packet networks is Fountain codes, also called rateless codes. The Fountain coding idea is proposed in [13] and followed by practical realizations such as LT codes [14], online codes [15], and Raptor codes [16]. Following the practical realizations, Fountain codes have gained attention in video streaming in recent years [17-19]. The main idea behind Fountain coding is to produce as many parity packets as needed on the fly. This approach is different from the general idea of FEC codes where channel encoding is performed for a fixed channel rate and all encoded packets are generated prior to transmission. The idea is proven to be efficient in [14] for large source data sizes, as in the case of video data, and it does not utilize retransmissions.

Due to a more intense prediction structure, stereoscopic video, the main focus of this work is more prone to packet losses compared to monoscopic video. Interdependent coding among views may result in quality distortion for both views if a packet from one view is lost. Even though FEC codes and optimal bit rate allocations are studied in depth for monoscopic video streaming, only few studies exist for stereoscopic video streaming [20]. In [20], stereoscopic video is layered using data partitioning, but an FEC method specific to stereoscopic video is not used. In our work, we aim at filling the gap in the literature on optimal error resilient streaming of stereoscopic video.

An overview of our proposed stereoscopic streaming system is presented in Figure 1. Initially, the scene of interest has to be captured with two cameras to obtain the raw stereoscopic video data. The video capture process is not in the scope of our work, thus we use publicly available raw video sequences. We encode the raw stereoscopic video data with an H.264-based multiview video encoder. We use the codec in stereoscopic mode and generate three layers which are denoted with the symbols $I, L$, and $R$. I-frames are the intracoded frames of the left view; L and R-frames are the intercoded frames of the left view and right view. The video encoder can encode each layer with different quantization parameters, thus with different bit rates $R_{I}, R_{L}$, and $R_{R}$. Due to lossy compression, the encoding process causes a distortion of $D_{e}$ in the video quality. After the stereoscopic encoder, we apply FEC to each layer separately where we use Raptor codes as the FEC scheme. The channel of interest in our system is a packet erasure channel of loss rate $p_{e}$, and the available bandwidth of the channel is $R_{C}$. We apply different protection rates $\rho_{I}, \rho_{L}$, and $\rho_{R}$ to each layer because they contribute differently to the video quality. After the lossy transmission, some of the packets are lost and Raptor decoder operates to recover the losses. However, some packets still may not be recovered, and the loss of these packets causes a distortion of $D_{\text {loss }}$ in the video quality. In this system, our goal is to obtain the optimal values of encoder bit rates $R_{I}, R_{L}$, and $R_{R}$ and protection rates $\rho_{I}, \rho_{L}$, and $\rho_{R}$ by minimizing the total distortion $D_{\text {tot }} \triangleq\left(D_{e}+D_{\text {loss }}\right)$. In order to execute the minimization, we obtain the analytical models of each part of our system. We start with the modeling of the RD curve of each layer of the stereoscopic video encoder. Then, we define the analytical model of the performance of Raptor codes. Finally, we estimate the distortion on the stereoscopic video quality caused by packet losses.

The organization of this paper is as follows. In Section 2, we describe the stereoscopic codec and define the layers of the stereoscopic video. In Section 3, we present the analytical model of the RD curve of the video encoder for each of the layers. In Section 4, we describe the Fountain codes and describe Raptor codes and their systematization. In Section 5, we define the analytical model of the Raptor coding performance curve. Then, in Section 6, we estimate the distortion caused by the loss of network abstraction layer (NAL) units. In Section 7, we minimize the total distortion, which includes both encoder and transmission distortions, in order to obtain the optimal encoder bit rates and UEP rates. We also evaluate the performance of the system and demonstrate its significant quality improvement on stereoscopic video. Finally, in Section 8, we conclude and state possible future work.

\section{Stereoscopic Codec}

The general structure of a stereoscopic encoder and decoder is given in Figure 2. In order to maintain backward compatibility to monoscopic decoders, left frames are encoded with prediction only from left frames, whereas right frames are predicted using both left and right frames. This enables standard monoscopic decoders to decode left frames. 

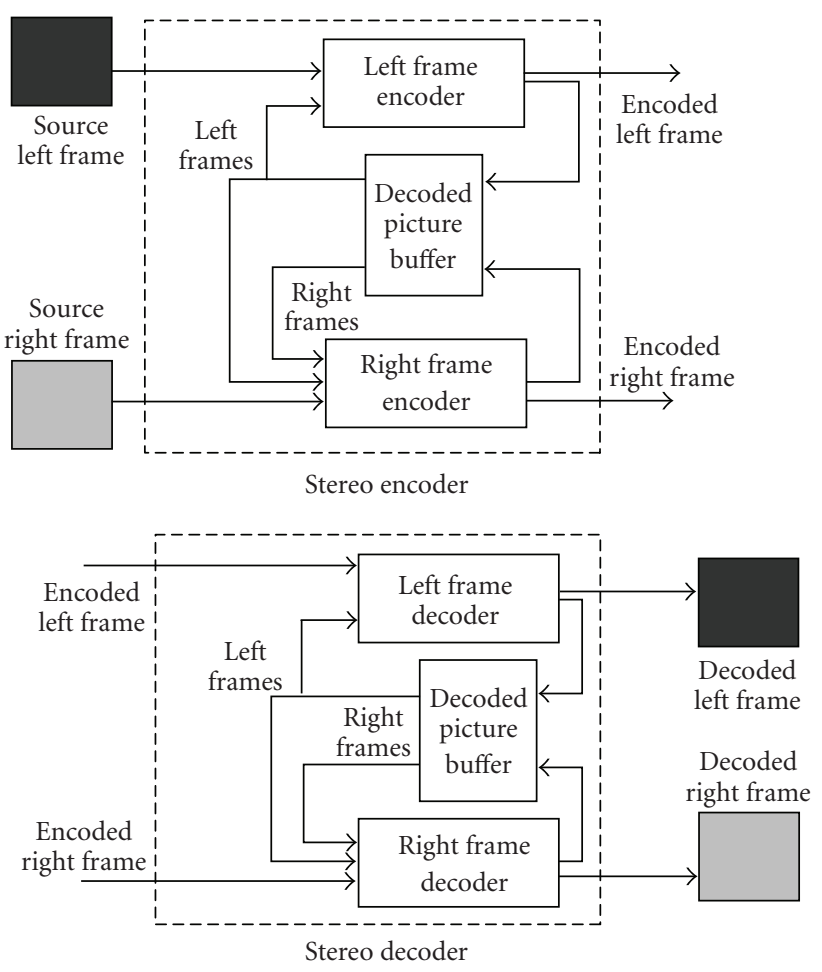

Figure 2: Stereoscopic encoder and decoder structure.

Any video codec with this basic structure can be used with the proposed streaming system in this work. Multiview extension of H.264 standard [21] (JMVM software) is one of the candidate codecs for this work. However, hierarchical Bpicture coding used in this codec increases the complexity. In order to decrease complexity and simplify decoding procedure, we have used [22], which is a multiview video codec based on H.264. This codec is an extension of standard H.264 with the structure given in Figure 2. In this codec, B frames are not supported. However, the results can easily be extended for JMVM codec.

The referencing structure of the codec in [22] is given in Figure 3, where we set the GOP size to 4 . Let $\mathbf{I}_{L}, \mathbf{P}_{L}$, and $\mathbf{P}_{R}$ denote the set of I-frames of left view, P-frames of left views, and P-frames of right views, respectively. The set of frames can be written in open form as $\mathbf{I}_{L}=\left\{I_{L 1}, I_{L 5}, \ldots\right\}$, $\mathbf{P}_{L}=\left\{P_{L 2}, P_{L 3}, \ldots\right\}, \mathbf{P}_{R}=\left\{P_{R 1}, P_{R 2}, \ldots\right\}$, where $L$ and $R$ indicate the frames of left and right video.

Although this coding scheme is not layered, frames are not equal in importance. We can classify the frames according to their contribution to the overall quality and use them as layers of the video. Since losing an I-frame causes large distortions due to motion/disparity compensation and error propagation, I-frames should be protected the most. Among P-frames, left frames are more important since they are referred by both left and right frames. According to this prioritization of the frames, we form three layers as shown in Figure 3. Layers can be coded with different quality (bit rate) by using either spatial scaling [23] or quantization. In this work, we use quantization parameter to adjust the quality of different layers.

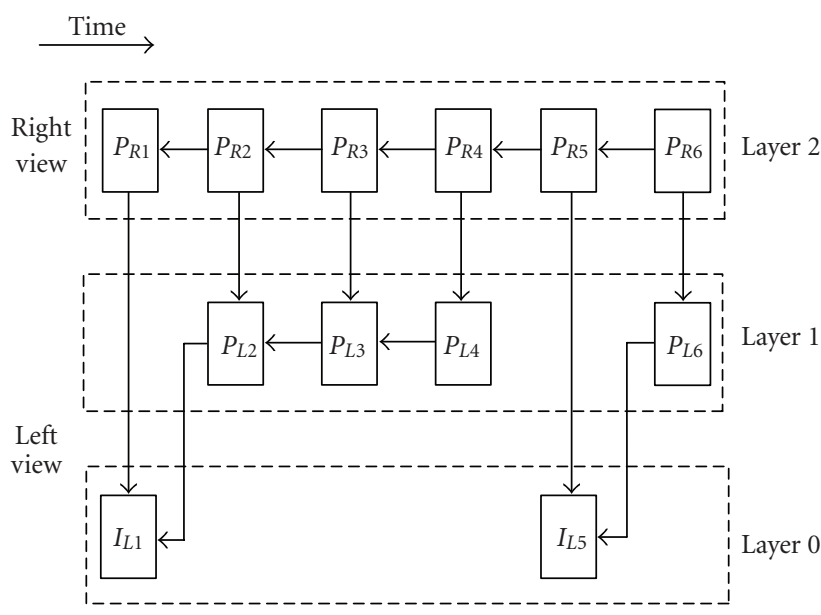

FIgURE 3: Layers of stereoscopic video and referencing structure.

In the case of slice losses in transmission, we employ different error concealment techniques for different layers in the decoder. For layer 0 , since there is no motion estimation, we use spatial concealment based on weighted pixel averaging [24]. For layer 1, we use temporal concealment. Colocated block from the previous layer- 1 frame is used in place of the lost block. For layer 2, we use temporal concealment but with a slight modification. In this case, colocated block can be taken either from previous layer-2 frame or from the layer-1 frame from the sametime index. Depending on the neighboring blocks motion vectors, appropriate frame is selected and colocated block from the selected frame is used in the place of the lost block.

\section{Analytical Model of the RD Curve of Encoded Stereoscopic Video}

In this section, we model the RD curve of stereoscopic video $\left(D_{e}\right.$ defined in Section 1$)$. The RD curve of video is widely used for optimal streaming purposes [5-8], which provides the optimal streaming bit rate for a given distortion in video quality and vice versa. In [25], a simple analytical RD curve model that can accurately approximate a wide range of monoscopic video sequences is presented. The model in [25] has the form

$$
D_{e}(R)=\frac{\theta}{R-R_{0}}+D_{0}
$$

where $D_{e}(R)$ is the mean-squared error (MSE) at the video encoder output at the encoding rate of $R$ bits/sec. There are 3 parameters to be solved which are $\theta, R_{0}$, and $D_{0}$. The parameters $R_{0}$ and $D_{0}$ do not correspond to any rate or distortion values and they are not initial values. At least, three samples of the RD curve are required to solve for the parameters.

The proposed analytical model in (1) can be used for each layer of video separately as stated in [25]. However, the model is not suitable for the cases when the layers are dependent. In our experiments, when we applied the analytical model 
in (1) separately to each one of our layers, we observed that the models were not accurate enough to approximate the RD curve. Thus, the analytical models had to be modified for dependent layers.

In our work, we have extended the analytical RD model of monoscopic video proposed in [25] to stereoscopic case and modified the model to handle the dependency among the layers. The structure of the layers of our stereoscopic codec is described in Section 2 and presented in Figure 3. The primary layer is layer 0 (I-frame) which consists of intraframes and it does not depend on any previous frames. Thus, the distortion of layer 0 only depends on the encoder bit rate of layer 0 . The second layer is layer 1 whose frames are coded dependent on previous frames of layer 1 and layer 0 . Thus, the distortion of layer 1 depends on the encoder bit rates of layer 1 and layer 0 . The third layer is layer 2 whose frames are coded dependent on previous frames of layer 2, layer 1 , and layer 0 . Thus, the encoder distortion of layer 2 depends on the encoder bit rates of all layers. We modeled the $\mathrm{RD}$ curves of each layer to include the stated dependencies.

3.1. RD Model of Layer 0. The RD curve model of layer 0 is given in (2). Layer 0 is encoded as an independent monoscopic video; hence, we model its RD curve using the same framework as in (1) and set the model as

$$
D_{e}^{I}\left(R_{I}\right)=\frac{\theta_{I}}{R_{I}-R_{0 I}}+D_{0 I}
$$

Here, $D_{e}^{I}\left(R_{I}\right)$ is the MSE coming from layer 0 when layer 0 is allocated a rate of $R_{I}$ bits/sec. The model parameters are $\theta_{I}, R_{0 I}$, and $D_{0 I}$ which have to be solved.

3.2. RD Model of Layer 1. The next analytical model is realized for layer 1 which consists of predicted frames of left view. As stated previously, the encoder distortion of layer 1 depends on the encoder bit rate of layer 1 and layer 0 . We modify the model in (1) to handle this dependency as

$$
D_{e}^{L}\left(R_{L}, R_{I}\right)=\frac{\theta_{L}}{R_{L}+c_{1} R_{I}-R_{0 L}}+D_{0 L}
$$

Here, $D_{e}^{L}\left(R_{L}, R_{I}\right)$ is the MSE coming from layer 1 when layer 1 and layer 0 are allocated the rates of $R_{L}$ and $R_{I}$ bits/sec, respectively. The model parameters are $\theta_{L}, c_{1}, R_{0 L}$, and $D_{0 L}$ which also have to be solved. The term $c_{1} R_{I}$ in the denominator is inserted to handle the dependency of the distortion of layer 1 to layer 0 , where the encoder bit rate of layer 0 is weighted with the parameter $c_{1}$.

3.3. RD Model of Layer 2. The last analytical model is realized for layer 2 which consists of the frames of right view. Since the distortion of layer 2 is dependent on all layers, the analytical model has to include the encoder bit rates of all layers. We modify the model in (1) to handle this dependency as

$$
D_{e}^{R}\left(R_{R}, R_{L}, R_{I}\right)=\frac{\theta_{R}}{R_{R}+c_{2} R_{I}+c_{3} R_{L}-R_{0 R}}+D_{0 R} .
$$

TABLE 1: Encoder RD curve parameters for "Rena" video.

\begin{tabular}{|c|c|c|c|c|c|}
\hline \multicolumn{3}{|l|}{ Layer 0} & $\begin{array}{c}\theta_{I} \\
2.978 \mathrm{e}+011\end{array}$ & $\begin{array}{c}R_{0 I} \\
10249\end{array}$ & $\begin{array}{c}D_{0 I} \\
120330\end{array}$ \\
\hline \multirow{2}{*}{ Layer 1} & & $c_{1}$ & $\theta_{L}$ & $R_{0 L}$ & $D_{0 L}$ \\
\hline & & 0.456 & $1.513 e+014$ & -23018 & 2209000 \\
\hline \multirow{2}{*}{ Layer 2} & $c_{2}$ & $c_{3}$ & $\theta_{R}$ & $R_{0 R}$ & $D_{0 R}$ \\
\hline & 0.333 & 0.235 & $1.496 e+014$ & 19482 & 6003200 \\
\hline
\end{tabular}

\begin{tabular}{lccccc}
\hline Layer 0 & & & $\theta_{I}$ & $R_{0 I}$ & $D_{0 I}$ \\
& & & $1.605 \mathrm{e}+011$ & 6050 & -289860 \\
\hline Layer 1 & & $c_{1}$ & $\theta_{L}$ & $R_{0 L}$ & $D_{0 L}$ \\
& & 0.616 & $3.483 \mathrm{e}+013$ & 51858 & 6142922 \\
\hline \multirow{2}{*}{ Layer 2 } & $c_{2}$ & $c_{3}$ & $\theta_{R}$ & $R_{0 R}$ & $D_{0 R}$ \\
& 0.308 & 0.086 & $4.535 \mathrm{e}+013$ & 50000 & 4056654 \\
\hline
\end{tabular}

TABLE 2: Encoder RD curve parameters for "Soccer" video.

Here, $D_{e}^{R}\left(R_{R}, R_{L}, R_{I}\right)$ is the MSE coming from layer 2 when layer 2, layer 1 , and layer 0 are allocated the rates of $R_{R}$, $R_{L}$, and $R_{I}$ bits/sec, respectively. The model parameters are $\theta_{R}, c_{2}, c_{3}, R_{0 R}$, and $D_{0 R}$, which also must be solved. The terms $c_{2} R_{I}$ and $c_{3} R_{L}$ in the denominator are inserted to handle the dependency of layer 2 to layer 0 and layer 1 , where the encoder bit rates of layer 0 and layer 1 are weighted with parameters $c_{2}$ and $c_{3}$.

3.4. Results on RD Modeling. In order to construct the $\mathrm{RD}$ curve models of stereoscopic videos, that is, to obtain the model parameters, we used curve fitting tools. In our work, we used the stereoscopic videos "Rena" and "Soccer" explained in Section 7.2 and obtained the RD curve models of these videos for the analytical models in (2) to (4). We used a general purpose nonlinear curve fitting tool which uses the Levenberg-Marquardt method with line search [26]. Before the curve fitting operation, we obtained many RD curve samples of the video by sweeping the quantization parameters of each layer from low to high quality. We obtained more RD samples than required in order to be able to observe the curve fitting performance. Then, we chose some of the RD samples and inserted into the curve fitting tool. The resulting analytical model parameters of the curve fit process are given in Tables 1 and 2 for the chosen videos. The parameters are in accordance with the properties of the videos. "Rena" has static background with moving objects and "Soccer" has a camera motion. Since the "Soccer" video has a camera motion, while encoding a right frame, correlation with the current left frame can be more than the previous right frame. This shows why the $c_{3}$ parameter of layer 2 of the "Soccer" video is high when compared with the results of the "Rena" video. 


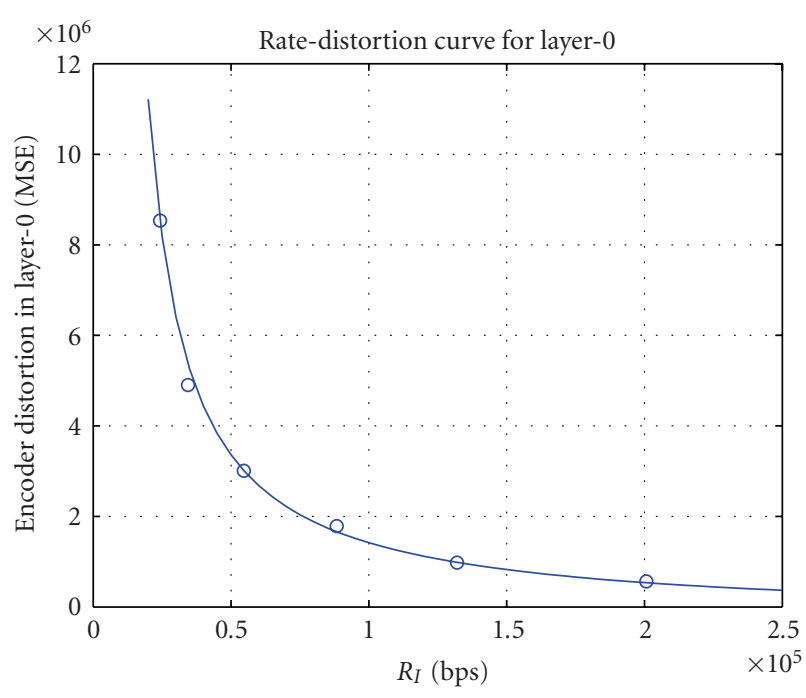

— Analytical model: $D_{e}^{I}\left(R_{I}\right)$

- RD samples

Figure 4: RD curve for layer 0 of the "Rena" video.

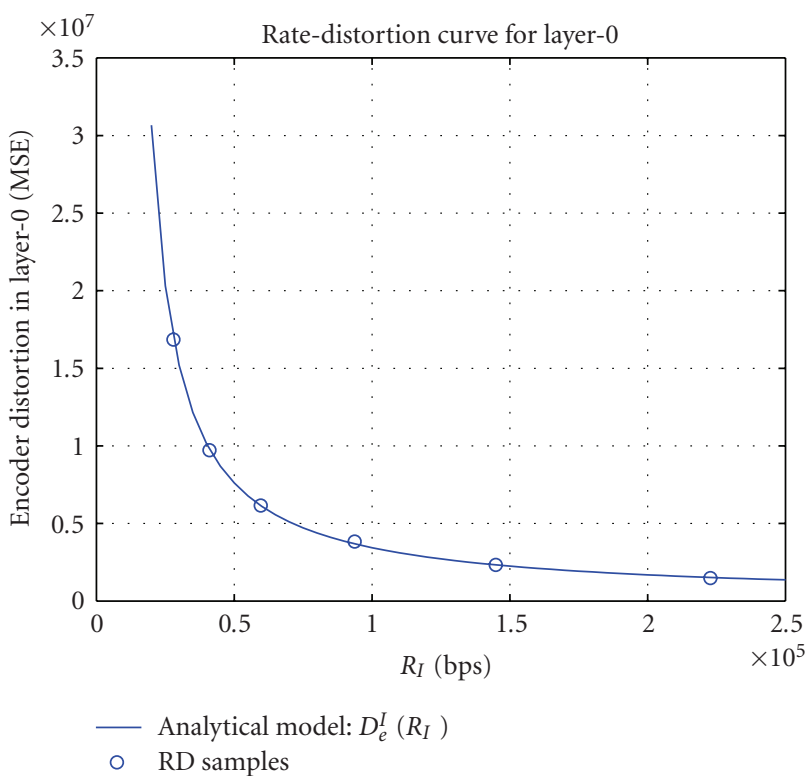

FIGURE 5: RD curve for layer 0 of the "Soccer" video.

In Figures 4 to 9, we present the results of analytical modeling of the RD curves. In Figures 4 and 5, we give the results for layer 0 , where the analytical models are constructed using the model in (2) with the corresponding parameters from Tables 1 and 2 . The RD samples correspond to the actual RD values obtained from the video encoder before the curve fitting process. Later, the results for layer 1 are presented in Figures 6 and 7 and those of layer 2 are presented in Figures 8 and 9. In the figures for layer 1 and 2, we present two cross-sections of the RD curves. The cross sections are obtained by fixing the encoder bit rates of the layers other than the corresponding layer of

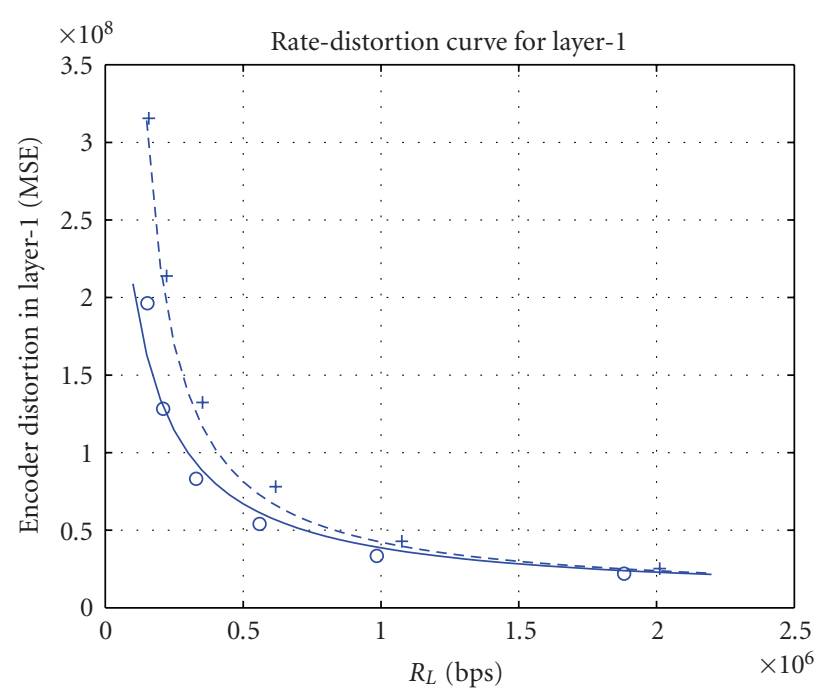

- Analytical model: $D_{e}^{L}\left(R_{L}, R_{I}=200.7 \mathrm{kbps}\right)$
○ $\quad$ RD samples, $R_{I}=200.7 \mathrm{kbps}$
-- Analytical model: $D_{e}^{L}\left(R_{L}, R_{I}=24.2 \mathrm{kbps}\right)$
$+\quad$ RD samples, $R_{I}=24.2 \mathrm{kbps}$

FIgURE 6: RD curve for layer 1 of the "Rena" video.

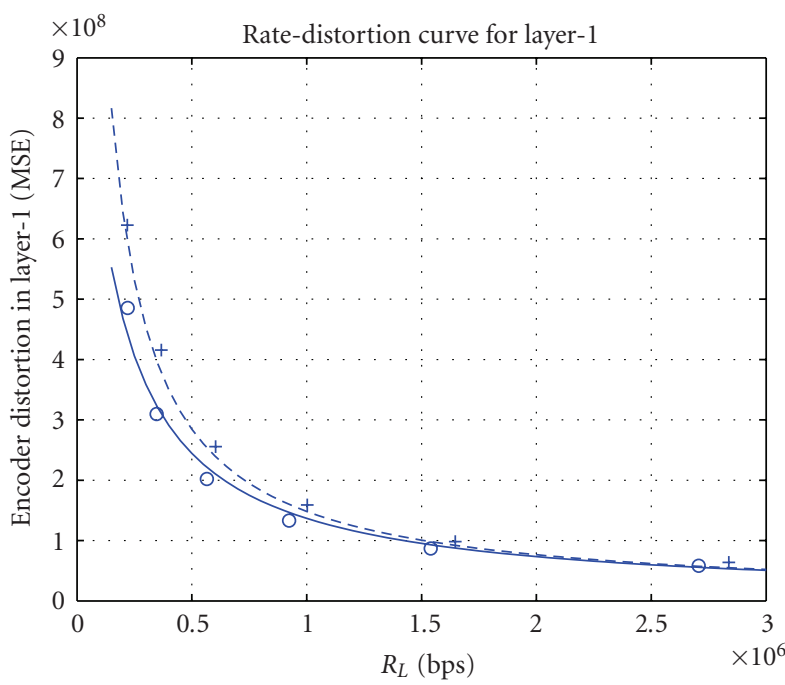

- Analytical model: $D_{e}^{L}\left(R_{L}, R_{I}=222.8 \mathrm{kbps}\right)$
$\circ \quad$ RD samples, $R_{I}=222.8 \mathrm{kbps}$
$--\quad$ Analytical model: $D_{e}^{L}\left(R_{L}, R_{I}=28 \mathrm{kbps}\right)$
$+\quad$ RD samples, $R_{I}=28 \mathrm{kbps}$

FIgURE 7: RD curve for layer 1 of the "Soccer" video.

interest. The average difference between analytical models and $\mathrm{RD}$ samples for the "Rena" video are 3.62\%, 7.60\%, and $9.19 \%$ for layer 0,1 , and 2 , respectively, and those of the "Soccer" video are $1.00 \%, 5.87 \%$, and $8.89 \%$. Thus, for both of the videos, which have different characteristics, satisfactory results are achieved where the analytical model approximates the RD samples accurately. 


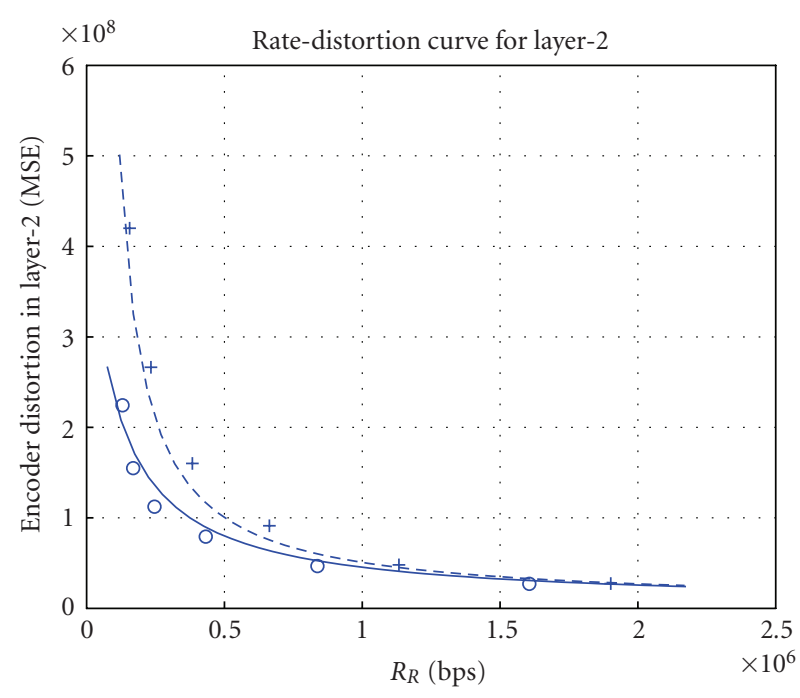

- Analytical model: $D_{e}^{L}\left(R_{R}, R_{L}=984.8 \mathrm{kbps}, R_{I}=200.7 \mathrm{kbps}\right)$

$\circ \quad$ RD samples, $R_{L}=984.8 \mathrm{kbps}, R_{I}=200.7 \mathrm{kbps}$

- - - Analytical model: $D_{e}^{L}\left(R_{R}, R_{L}=157.9 \mathrm{kbps}, R_{I}=24.2 \mathrm{kbps}\right)$

$+\quad$ RD samples, $R_{L}=157.9 \mathrm{kbps}, R_{I}=24.2 \mathrm{kbps}$

Figure 8: RD curve for layer 2 of the "Rena” video.

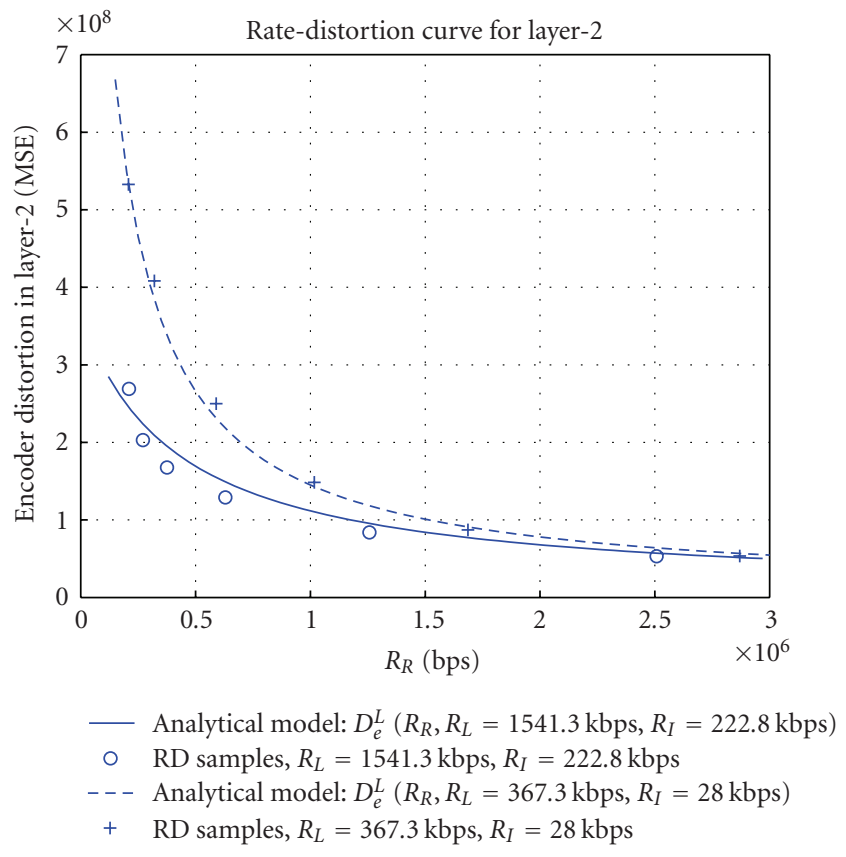

FIgURE 9: RD curve for layer 2 of the "Soccer" video.

\section{Raptor Codes}

In our work, we use Raptor codes [16] as the FEC scheme to protect the encoded stereoscopic video data from the packet losses during transmission. We choose Raptor codes due to their low complexity and ease of employability on packet networks. Raptor codes are the most recent practical realization of Fountain codes [13]. Fountain codes, also called rateless codes, are a novel class of FEC codes where

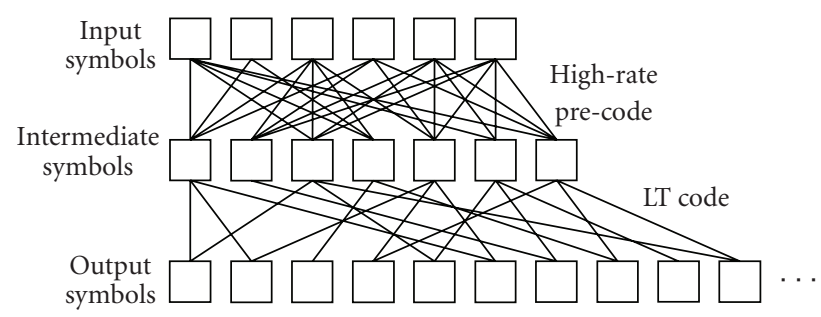

FIGURE 10: Representation of Raptor encoder.

as many parity packets as needed are generated on the fly. Fountain codes are low complexity channel codes providing reliability, low latency, and loss rate adaptability. There are many practical realizations of fountain codes such as Luby transform (LT) codes [14], online codes [15], and the most recent one being Raptor codes. In all of the Fountain coding schemes the original data is divided into $k$ packets (source packets) denoted as input symbols. The encoded packets (transmitted packets) are denoted as output symbols. An ideal fountain encoder can generate potentially limitless output symbols in linear complexity and an ideal fountain decoder can reconstruct the original data in linear complexity if any $k(1+\varepsilon)$ of the output symbols are received, where $\varepsilon$ goes to zero as $k$ increases.

Raptor codes are an extension of LT codes and their encoding structure is represented in Figure 10. They have two consecutive channel encoders, where the precode is a high-rate FEC code and the outercode is an LT code. Input symbols are the data units of the original source data. An input symbol can be a bit or a symbol composed of $s$ bits. In our work, each NAL unit generated by the stereoscopic video encoder corresponds to an input symbol. The precode generates intermediate symbols which are not transmitted but are used as an intermediate step to generate the transmitted output symbols. The precode is presented to reduce the overhead of LT codes. LDPC codes [27] are the most commonly used FEC codes as the precode on Raptor codes.

In the following, we define the input output relations for the Raptor coder in our work. For now, assume that we are given the parity ratio $\rho$ and the bit rate of encoded video $R$. Let $N_{\text {bits }}$ denote the number of bits in a NAL unit, then the number of input symbols can be defined as $N_{i}=$ $R / N_{\text {bits }}$, and the number of output symbols can be calculated as $N_{o}=(1+\rho) N_{i}$. The Raptor encoder forms $N_{o}$ output symbols which are linear combinations of the input symbols chosen from a degree distribution. Details on the degree distributions are given in [16]. The Raptor decoder receives $N_{r}$ out of $N_{o}$ of these output symbols after lossy transmission. Any algorithm that solves for the input symbols using these $N_{r}$ output symbols is a Raptor decoder.

Similar to any linear block code, Raptor codes can be systematic or nonsystematic. In systematic codes, the transmitted symbols consist of the original data symbols and the parity symbols, whereas in the nonsystematic case the original data symbols are transformed into new symbols for transmission. The access to original data is beneficial in 
video transmission applications since $100 \%$ reliability is not obliged. When the video data is encoded with systematic channel codes, even if the channel decoder cannot decode all of the input symbols, the video decoder can use error concealment techniques to approximate the lost symbols of the video. In our work, we use systematic Raptor codes as the FEC scheme. For our systematic Raptor coding implementation, we use a practical and low-complexity scheme described in [28].

\section{Analytical Modeling of the Performance Curve of Raptor Codes}

In this section, we model the performance curve of Raptor codes. The performance curve of Raptor codes is defined as the graph that represents the average number of undecoded input symbols versus the number of received output symbols. Thus, we aim at obtaining the analytical model of the residual number of lost packets after the channel decoder.

5.1. Performance Curve Model. We propose a heuristic analytical model of the performance curve of Raptor codes which is going to be used for the derivation of optimal parity packet allocation to layers in Section 7 in the end-to-end distortion minimization. We define the analytical model as

$$
N_{u}\left(N_{i}, N_{r}, \rho\right)= \begin{cases}N_{i}-\frac{N_{r}}{(1+\rho)}, & N_{r} \leq N_{i}, \\ N_{i} \frac{\rho}{(1+\rho)} 2^{\left(N_{i}-N_{r}\right)}, & N_{r}>N_{i} .\end{cases}
$$

In (5), $N_{u}\left(N_{i}, N_{r}, \rho\right)$ is the analytical model of the number of undecoded input symbols which is a function of $N_{i}$, $N_{r}$, and $\rho$. In order to form the model, we investigate the performance curve in two separate regions; first, in the region with the number of received symbols less than or equal to number of input symbols and, second, in the remaining region. In the first region of the model, we assume that the Raptor decoder cannot decode any lost symbols other than the received systematic symbols. whereas, in the second region, an exponential decrease in the number of undecoded symbols is assumed.

5.2. Results on the Performance Curve Modeling. In Figure 11, the actual performance curve and the analytical model are presented for $N_{i}=100$ and $\rho=0.5$. In Figure 12, we provide the curves zoomed around $N_{r}=100$ for the curves given in Figure 11. In Figures 13 and 14, results with different parity ratios and different number of input symbols are presented. In the figures, we provide the actual performance curve and the analytical model for comparison. We obtain the actual performance curve as follows. Initially, for given $N_{i}$ and $\rho,(1+\rho) N_{i}$ output symbols are created as described in [28]. Then, randomly $N_{r}$ output symbols are selected and inserted to Raptor decoder and the number of undecoded input symbols are recorded. For each value of $N_{r}$ (1 to $\left.(1+\rho) N_{i}\right)$, this process is repeated for 200 times and the number of undecoded symbols are averaged to obtain the

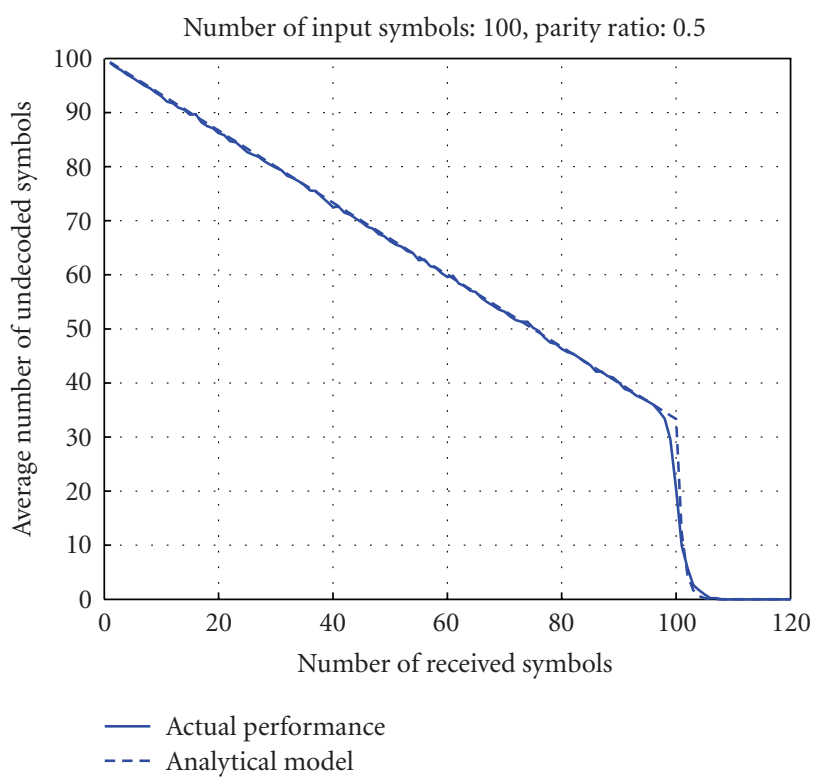

Figure 11: Performance curve of Raptor coding, $N_{i}=100, \rho=0.5$.

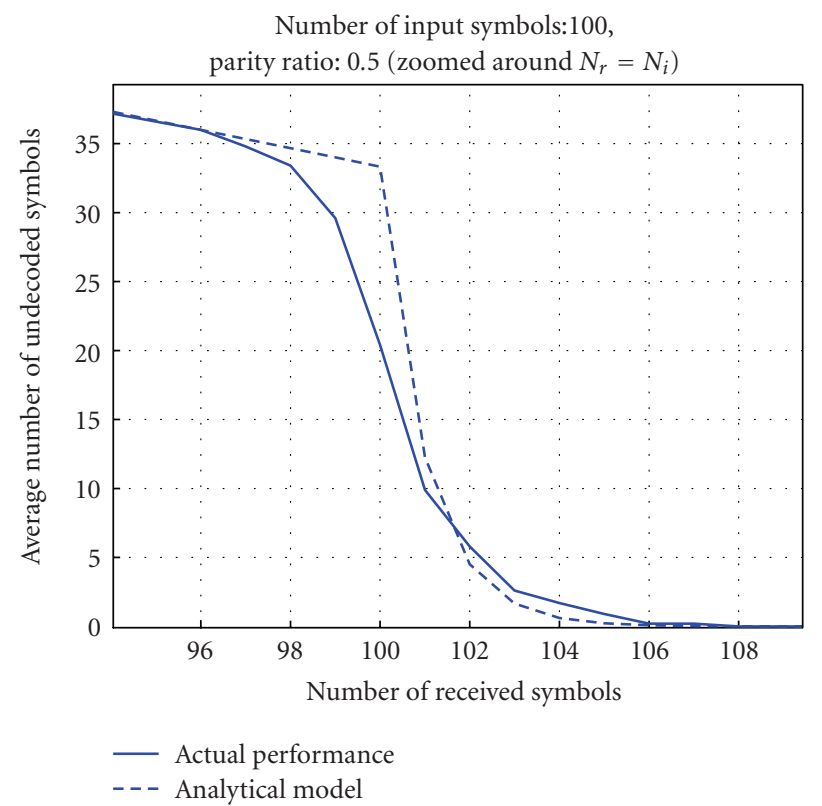

Figure 12: Performance curve of Raptor coding (zoomed around $\left.N_{r}=N_{i}\right), N_{i}=100, \rho=0.5$.

actual performance. We obtained the analytical model with (5) by plotting $N_{u}$ versus $N_{r}$ for given $N_{i}$ and $\rho$. As observed from the figures, the analytical model approximates the performance curve of Raptor codes accurately.

\section{Estimation of Transmission Distortion}

In this section, our aim is to estimate the residual loss distortion in video remaining after the Raptor decoder and stereoscopic video decoder $\left(D_{\text {loss }}\right.$ defined in Section 1$)$. In the 


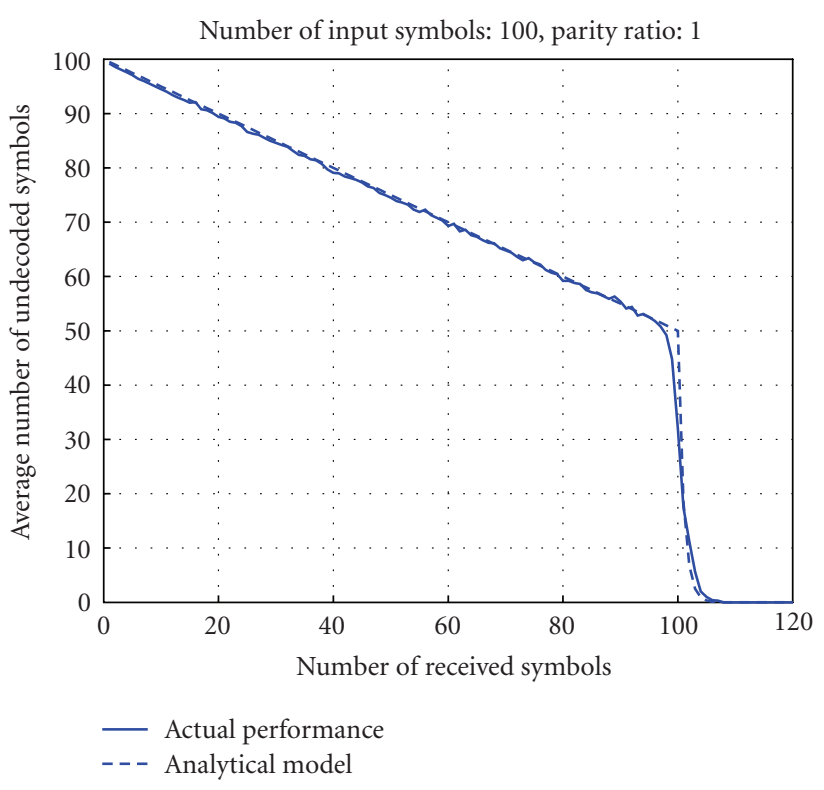

FIGURE 13: Performance curve of Raptor coding, $N_{i}=100, \rho=1.0$.

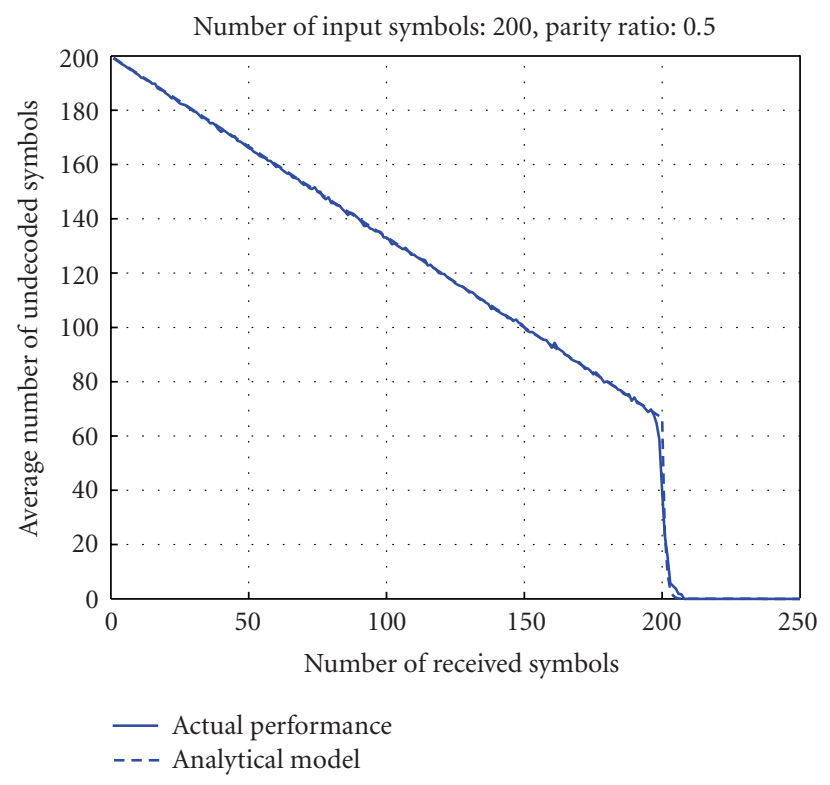

Figure 14: Performance curve of Raptor coding, $N_{i}=200, \rho=0.5$.

following sections, we explain the estimation of residual loss distortion step by step.

6.1. Lossy Transmission. The channel of interest in our work is PEC as mentioned previously. During the transmission of stereoscopic video layers from PEC, NAL units are lost with probability $p_{e}$. In the remaining part of our work, for simplicity, $X$ will represent the layer denotations $I, L$, and $R$. As explained in the system overview in Section 1, we have three layers of video with source bit rate $R_{X}$ which are Raptor encoded separately with inserted parity rate $\rho_{X}$. Thus,
$N_{i}^{X}\left(1+\rho_{X}\right)$ output symbols are created and transmitted for each layer. After lossy transmission, the number of received output symbols in Raptor decoder can be calculated as

$$
N_{r}^{X}=N_{i}^{X}\left(1+\rho_{X}\right)\left(1-p_{e}\right) .
$$

Here, we use the average loss probability for simplified modeling purposes only. The experimental results in Section 7.2 reflect the actual distortions over lossy channels, where a single packet is lost with probability $P_{e}$.

6.2. Reconstruction of Input Symbols in Raptor Decoder. After receiving $N_{r}^{X}$ output symbols Raptor decoder operates to solve for the input symbols. We use the model of the performance curve of Raptor codes to obtain the average number of undecoded input symbols using (5). The average number of undecoded input symbols (the residual number of lost NAL units) can be calculated as

$$
N_{u}^{X}=N_{u}\left(N_{i}^{X}, N_{r}^{X}, \rho_{X}\right) .
$$

6.3. Propagation of Lost NAL Units in Stereoscopic Video Decoder. Due to the recursive structure of the video codec, the distortion of an NAL unit loss not only causes distortion in the corresponding frame, but it also propagates to subsequent frames in the video. Initially, since each NAL unit contains a specific number of macroblocks (MBs), we estimate the distortion in a frame when a single $M B$ is lost. The distortion is calculated after error concealment techniques, explained in Section 2, are applied for the lost $\mathrm{MB}$. Then, we calculate the average propagated distortion of a single $\mathrm{MB}$ and, consequently, an NAL unit.

In [25], a model for distortion propagation is proposed, where the propagated error energy (distortion) at frame $t$ after a loss at frame 0 is given as

$$
\sigma_{u}^{2}(t)=\frac{\sigma_{u 0}^{2}}{1+\gamma t} .
$$

Here, $\sigma_{u 0}^{2}$ is the average distortion per lost unit, and $\gamma$ is the leakage factor which describes the efficiency of the loop filtering in the decoder to remove the introduced error $(0<\gamma<1)$. We assume $\gamma \approx 0$ which results in worst case propagation, where the distortion propagates equally to all subsequent frames $\left(\sigma_{u}^{2}(t)=\sigma_{u 0}^{2}\right)$. In the following sections, we calculate the propagated NAL unit loss distortion for each layer separately, where we set MBs as the video unit.

6.3.1. NAL Unit Loss from Layer 0. The expression in (9) gives the average distortion of spatial error concealment when a lost $\mathrm{MB}$ is concealed by the average of its neighboring MBs. In (9), $S_{\mathrm{MB}}, \mathrm{MB}_{i}, S_{\mathrm{MB}, i}, N_{i}^{\prime}$, and $N_{\mathrm{MB}}^{I}$ represent the set of macroblocks, the $i$ th macroblock, the set of $i$ th MB's neighbors, the number of neighbors of $i$ th $\mathrm{MB}$, and the number of MBs of layer 0 , respectively. $I_{I}(x, y, 0)$ denotes the pixel in position $(x, y)$ of the intraframe of layer 0 . Layer 0 consists of a single intraframe, thus only spatial error 


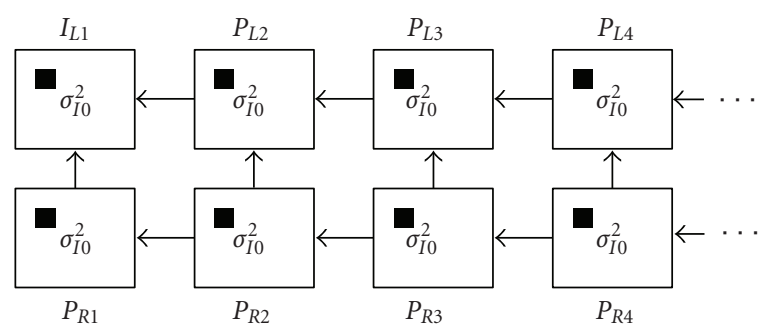

FIgure 15: Propagation of an MB loss from I-frame.

concealment can be used due to intracoding as described in Section 2:

$$
\begin{aligned}
& \sigma_{I 0}^{2} \\
& =\frac{1}{N_{\mathrm{MB}}^{I}} \sum_{k \in S_{\mathrm{MB}}}\left[\sum_{x, y \in \mathrm{MB}_{k}}\left(I_{I}(x, y, 0)-\sum_{x^{\prime}, y^{\prime} \in \mathrm{MB}_{k}^{\prime}} I_{I}\left(x^{\prime}, y^{\prime}, 0\right) / N_{k}^{\prime}\right)^{2}\right] .
\end{aligned}
$$

In Figure 15, the propagation of an MB loss in an I-frame is demonstrated. The black box in the frame $I_{L 1}$ represents a possible loss in the I-frame. The loss causes a distortion of $\sigma_{I 0}^{2}$ as calculated in (9) for the frame $I_{L 1}$. The loss propagates to all subsequent frames with equal distortion on the average since both L-frames and R-frames refer initially to the Iframe. If we denote the GOP size as $\mathrm{T}$, then the average of total propagated loss distortion when an $\mathrm{MB}$ is lost from layer 0 can be calculated as

$$
D_{\mathrm{MB} \text { prop }}^{I}=2 \mathrm{~T} \sigma_{I 0}^{2}
$$

In order to calculate the average distortion of losing an NAL unit from layer $0\left(D_{\mathrm{NAL} \text { loss }}^{I}\right)$, we have to calculate the average number of MBs in a NAL unit. Let $N_{\mathrm{MB}}^{I}$ denote the number of MBs in layer 0 . Then, $D_{\mathrm{NAL} \text { loss }}^{I}$ can be calculated as

$$
D_{\mathrm{NAL} \text { loss }}^{I}=\left(\frac{N_{\mathrm{MB}}^{I}}{N_{i}^{I}}\right) \cdot D_{\mathrm{MB} \text { prop }}^{I} \cdot
$$

6.3.2. NAL Unit Loss from Layer 1. The expression in (12) gives the average distortion of temporal error concealment when a lost NAL unit is concealed from the previous frame of layer 1. In (12), $N_{\mathrm{MB}}^{L}$ and T represent the number of MBs of layer 1 and GOP size, respectively. $I_{L}(x, y, i)$ denotes the pixel in position $(x, y)$ of $i$ th frame of layer 1 . Layer 1 consists of predicted frames of left view. In our stereoscopic codec, we used temporal error concealment for layer 1 as described in Section 2:

$$
\sigma_{L 0}^{2}=\frac{(1 /(T-1)) \sum_{i=1}^{T-1} \sum_{x, y}\left[I_{L}(x, y, i)-I_{L}(x, y, i-1)\right]^{2}}{N_{\mathrm{MB}}^{L}} .
$$

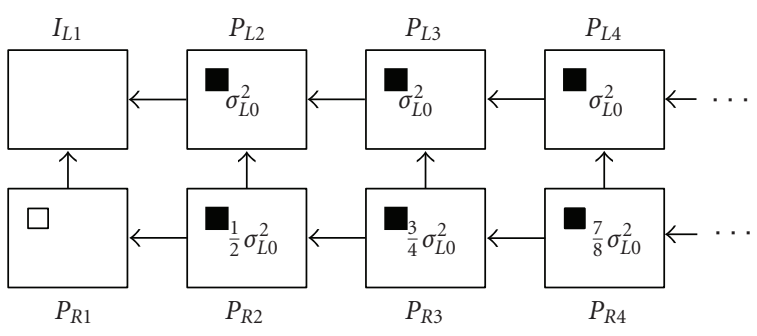

Figure 16: Propagation of an MB loss from L-frame.

In Figure 16, the propagation of an MB loss in an L-frame is demonstrated. The black box in the frame $P_{L 2}$ represents a possible loss in the L-frame. The loss causes a distortion of $\sigma_{L 0}^{2}$ as calculated in (12) for the frame $P_{L 2}$. The loss propagates to all subsequent L-frames with equal distortion since each L-frame refers to the previous L-frame. Let $m$ denote the frame index of loss in a GOP, then the average propagated loss to L-frames can be calculated as

$$
\frac{1}{T-1} \sum_{m=1}^{T-1}(T-m) \sigma_{L 0}^{2}
$$

The MB loss also propagates to R-frames. However, Rframes not only refer to current L-frames but also previous R-frames. Due to this fact, the distortion in $P_{R 2}$ can be calculated as $\sigma_{L 0}^{2} / 2$ using the previous undistorted MB (white box in $\left.P_{R 1}\right)$. In the frame $P_{R 3}$ the propagated distortion can be calculated as $\left(\sigma_{L 0}^{2} / 2+\sigma_{L 0}^{2}\right) / 2=(3 / 4) \sigma_{L 0}^{2}$. In the subsequent frames, the propagated distortion is calculated similarly as shown in Figure 16. The average of total propagated distortion in an R-frame caused by the loss of an L-frame $\mathrm{MB}$ can be calculated as

$$
\frac{1}{T-1} \sum_{m=1}^{T-1} \sum_{n=1}^{T-m}\left(\left(1-\frac{1}{2^{n}}\right) \sigma_{L 0}^{2}\right) \text {. }
$$

Thus, the average of total propagated distortion when an $\mathrm{MB}$ is lost from layer 1 can be calculated as

$$
D_{\mathrm{MB} \text { prop }}^{L}=\frac{1}{T-1} \sum_{m=0}^{T-2} \sum_{n=0}^{m}\left(\left(2-\frac{1}{2^{n+1}}\right) \sigma_{L 0}^{2}\right) .
$$

In order to calculate the average distortion of losing an NAL unit from layer 1 ( $\left.D_{\text {NAL loss }}^{L}\right)$, we have to calculate the average number of MBs in an NAL unit. Let $N_{\mathrm{MB}}^{L}$ denote the number of MBs in layer 1 . Then, $D_{\mathrm{NAL} \text { loss }}^{L}$ can be calculated as

$$
D_{\mathrm{NAL} \text { loss }}^{L}=\left(\frac{N_{\mathrm{MB}}^{L}}{N_{i}^{L}}\right) \cdot D_{\mathrm{MB} \text { prop }}^{L}
$$

6.3.3. NAL Unit Loss from Layer 2. The expression in (17) gives the average distortion of temporal error concealment when a lost NAL unit is concealed from the frames of layer 2 and layer 1. In (17), $N_{\mathrm{MB}}^{R}$ and $T$ represent the number of MBs of layer 2 and GOP size, respectively. $I_{R}(x, y, i)$ denotes the 


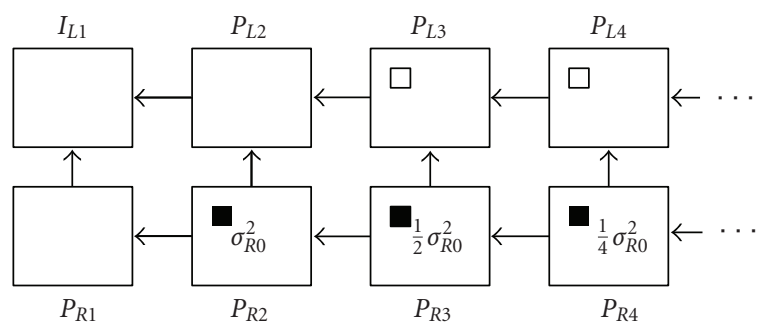

Figure 17: Propagation of an MB loss from R-frame.

pixel in position $(x, y)$ of $i$ th frame of layer 2. Layer 2 consists of predicted frames of right view. In our stereoscopic codec, we used temporal error concealment for layer 2, where the frames are referred to previous layer 2 and current layer 1 frames as described in Section 2:

$$
\begin{aligned}
\sigma_{R 0}^{2}= & \frac{\sum_{x, y}\left[I_{L}(x, y, 0)-I_{R}(x, y, 0)\right]^{2}}{(T-1) N_{\mathrm{MB}}^{R}} \\
& +\frac{\sum_{i=1}^{T-1} \sum_{x, y}\left[Q-I_{R}(x, y, i)\right]^{2}}{(T-1) N_{\mathrm{MB}}^{R}},
\end{aligned}
$$

where $Q=\left(\left(I_{R}(x, y, i-1)+I_{L}(x, y, i)\right) / 2\right)$.

In Figure 17, the propagation of an MB loss in an Rframe is demonstrated. The black box in the frame $P_{R 2}$ represents a possible loss in the R-frame. The loss in an Rframe propagates only to the subsequent R-frames. A loss in the frame $P_{R 2}$ creates a distortion of $\sigma_{R 0}^{2}$ as calculated in (17). In frame $P_{R 3}$, the propagation distortion can be calculated as $\sigma_{R 0}^{2} / 2$ using the undistorted $\mathrm{MB}$ in the L-frame (white box in $\left.P_{L 3}\right)$. In each of the following R-frames, the propagated distortion is the half of the previous R-frame. Thus, the average of total propagated distortion when an $\mathrm{MB}$ is lost from layer 2 can be calculated as

$$
D_{\mathrm{MB} \text { prop }}^{R}=\sum_{m=0}^{T-1} \frac{1}{T} \sum_{n=0}^{m}\left(\frac{1}{2^{n}} \sigma_{R 0}^{2}\right) .
$$

In order to calculate the average distortion of losing an NAL unit from layer 2 ( $D_{\mathrm{NAL} \text { loss }}^{R}$ ), we have to calculate the average number of MBs in an NAL unit. Let $N_{\mathrm{MB}}^{R}$ denote the number of MBs in layer 2. Then, $D_{\mathrm{NAL}}^{R}$ loss can be calculated as

$$
D_{\mathrm{NAL} \text { loss }}^{R}=\left(\frac{N_{\mathrm{MB}}^{R}}{N_{i}^{R}}\right) \cdot D_{\mathrm{MB} \text { prop }}^{R} \text {. }
$$

6.4. Calculation of Residual Loss Distortion. In this part, we calculate the average transmission distortion after Raptor decoder and stereoscopic video decoder. Let $D_{\text {loss }}^{X}$ denote the residual transmission distortion. In (20), we calculate $D_{\text {loss }}^{X}$ by multiplying the number of undecoded input symbols with the average distortion of losing an NAL unit:

$$
D_{\text {loss }}^{X}\left(R_{X}, \rho_{X}, p_{e}\right)=N_{u}\left(N_{i}^{X}, N_{r}^{X}, \rho_{X}\right) \cdot D_{\text {NAL loss }}^{X} \text {. }
$$

Here, we use the assumption that the NAL unit losses are uncorrelated which is met for low number of losses after the Raptor decoder. Thus, the accuracy of the model may reduce for high loss rates.

\section{End-to-End Distortion Minimization and Performance Evaluation}

As the last part of our system, we minimize the total end-toend distortion to find the optimal encoder bit rates and UEP rates and evaluate the performance of the system. We present the minimization as

$$
\begin{aligned}
& \min _{\left(R_{I}, R_{L}, R_{R}, \rho_{I}, \rho_{L}, \rho_{R}\right)} D_{\text {tot }} \\
& \text { s.t. }\left(1+\rho_{I}\right) R_{I}+\left(1+\rho_{L}\right) R_{L}+\left(1+\rho_{R}\right) R_{R}=R_{C} .
\end{aligned}
$$

The minimization aims at obtaining the optimal encoder bit rates $R_{I}, R_{L}$, and $R_{R}$, and optimal parity ratios $\rho_{I}, \rho_{L}$, and $\rho_{R}$ for given $p_{e}$ and $R_{C}$. The constraint ensures that the final bit rate satisfies a total transmission bandwidth of $R_{C}$ including both the encoder bit rates and protection data bit rates. In (22), we present the calculation of $D_{\text {tot }}$ where $D_{e}^{I}(\cdot)$, $D_{e}^{L}(\cdot)$, and $D_{e}^{R}(\cdot)$ are the encoder distortions defined in (2), (3), and (4), and $D_{\text {loss }}^{I}(\cdot), D_{\text {loss }}^{L}(\cdot)$, and $D_{\text {loss }}^{R}(\cdot)$ are the residual loss distortions defined in $(20)$ :

$$
\begin{aligned}
& D_{\mathrm{tot}}= \frac{1}{3}\left[D_{e}^{R}\left(R_{R}, R_{L}, R_{I}\right)+D_{\text {loss }}^{R}\left(R_{R}, r_{r}, p_{e}\right)\right] \\
&+\frac{2}{3}\left[D_{e}^{I}\left(R_{I}\right)+D_{e}^{L}\left(R_{L}, R_{I}\right)+D_{\text {loss }}^{I}\left(R_{I}, \rho_{I}, p_{e}\right)\right. \\
&\left.\quad+D_{\text {loss }}^{L}\left(R_{L}, \rho_{L}, p_{e}\right)\right] .
\end{aligned}
$$

Total distortion in left and right frames is weighted to handle the objective stereoscopic video quality as stated in [29]. The weighting parameters in [29] are found by least squares fitting of the subjective results with the distortion values. In [29], there are three parameters used for coding, number of layers, quantization parameter for left view, and temporal scaling. In our codec, we are only using quantization parameter for adjusting the bit rates. Although both codecs are not the same, they are both extensions of H.264 JM and JSVM softwares. So, the distortions become similar if we consider only the case where quantization parameter is used to adjust the bit rates. Also, subjective results for our codec with temporal and spatial scaling can be found in [24], where we have similar results given in [29].

7.1. Results on the Minimization of End-to-End Distortion. We solve the minimization in (21) by a general purpose minimization tool which uses sequential quadratic programing where the tool solves a quadratic programing at each iteration as described in [30]. In our work, we obtain the optimal encoder bit rates and parity ratios for $P_{e} \in\{0.03$, $0.05,0.1,0.2\}$ and $R_{C} \in\{500,750,1000,1500,2000,2500$ (kbps) $\}$ for "Rena" video and $R_{C} \in\{1000,1500,2000,2500$, 3000, 3500 (kbps) \} for "Soccer" video. Thus, we perform 24 optimizations per video using (21).

In Tables 3 and 4, the optimal encoder bit rates and protection rates for the proposed method are given for the "Rena" and "Soccer" stereoscopic videos for $p_{e}=0.10$. The encoder bit rates of the right view are lower than that of the left view, which is caused by the unequal weighting in the total distortion expression in (22). The protection rate of 
TABle 3: Video encoder bit rates and Raptor encoder protection rates for "Rena" video.

\begin{tabular}{|c|c|c|c|c|c|c|c|c|c|c|c|c|}
\hline \multicolumn{13}{|c|}{$P_{e}=0.1$} \\
\hline \multirow[t]{3}{*}{$R_{C}(\mathrm{Kbps})$} & \multirow{2}{*}{\multicolumn{3}{|c|}{$\begin{array}{c}\text { Encoder bit rates (Kbps) } \\
\text { (optimal) }\end{array}$}} & \multicolumn{9}{|c|}{ Protection rates } \\
\hline & & & & \multicolumn{3}{|c|}{ Proposed (optimal) } & \multicolumn{3}{|c|}{ EEP } & \multicolumn{3}{|c|}{ Protect-L } \\
\hline & $R_{I}$ & $R_{L}$ & $R_{R}$ & $\rho_{I}$ & $\rho_{L}$ & $\rho_{R}$ & $\rho_{I}$ & $\rho_{L}$ & $\rho_{R}$ & $\rho_{I}$ & $\rho_{L}$ & $\rho_{R}$ \\
\hline 500 & 33.5 & 216.6 & 169.8 & 0.489 & 0.177 & 0.147 & 0.190 & 0.190 & 0.190 & 0.320 & 0.320 & 0.000 \\
\hline 750 & 51.5 & 337.8 & 250.7 & 0.389 & 0.158 & 0.143 & 0.172 & 0.172 & 0.172 & 0.282 & 0.282 & 0.000 \\
\hline 1000 & 69.6 & 460.0 & 332.2 & 0.332 & 0.148 & 0.139 & 0.160 & 0.160 & 0.160 & 0.260 & 0.260 & 0.000 \\
\hline 1500 & 106.0 & 705.6 & 496.0 & 0.270 & 0.138 & 0.133 & 0.147 & 0.147 & 0.147 & 0.237 & 0.237 & 0.000 \\
\hline 2000 & 142.4 & 951.9 & 660.3 & 0.236 & 0.132 & 0.129 & 0.140 & 0.140 & 0.140 & 0.224 & 0.224 & 0.000 \\
\hline 2500 & 178.9 & 1198.7 & 824.8 & 0.215 & 0.128 & 0.127 & 0.135 & 0.135 & 0.135 & 0.216 & 0.216 & 0.000 \\
\hline
\end{tabular}

TABLE 4: Video encoder bit rates and Raptor encoder protection rates for "Soccer" video.

\begin{tabular}{|c|c|c|c|c|c|c|c|c|c|c|c|c|}
\hline \multicolumn{13}{|c|}{$P_{e}=0.1$} \\
\hline \multirow[t]{3}{*}{$R_{C}(\mathrm{Kbps})$} & \multirow{2}{*}{\multicolumn{3}{|c|}{$\begin{array}{c}\text { Encoder bit rates (Kbps) } \\
\text { (optimal) }\end{array}$}} & \multicolumn{9}{|c|}{ Protection rates } \\
\hline & & & & \multicolumn{3}{|c|}{ Proposed (optimal) } & \multicolumn{3}{|c|}{ EEP } & \multicolumn{3}{|c|}{ Protect-L } \\
\hline & $R_{I}$ & $R_{L}$ & $R_{R}$ & $\rho_{I}$ & $\rho_{L}$ & $\rho_{R}$ & $\rho_{I}$ & $\rho_{L}$ & $\rho_{R}$ & $\rho_{I}$ & $\rho_{L}$ & $\rho_{R}$ \\
\hline 1000 & 68.4 & 543.0 & 245.9 & 0.349 & 0.147 & 0.156 & 0.166 & 0.166 & 0.166 & 0.233 & 0.233 & 0.000 \\
\hline 1500 & 96.0 & 833.8 & 373.7 & 0.294 & 0.136 & 0.145 & 0.151 & 0.151 & 0.151 & 0.211 & 0.211 & 0.000 \\
\hline 2000 & 123.7 & 1125.3 & 501.9 & 0.260 & 0.130 & 0.138 & 0.142 & 0.142 & 0.142 & 0.199 & 0.199 & 0.000 \\
\hline 2500 & 151.3 & 1417.2 & 630.3 & 0.238 & 0.127 & 0.134 & 0.137 & 0.137 & 0.137 & 0.192 & 0.192 & 0.000 \\
\hline 3000 & 179.0 & 1709.3 & 758.7 & 0.222 & 0.125 & 0.131 & 0.133 & 0.133 & 0.133 & 0.186 & 0.186 & 0.000 \\
\hline 3500 & 206.6 & 2001.6 & 887.3 & 0.209 & 0.123 & 0.128 & 0.131 & 0.131 & 0.131 & 0.183 & 0.183 & 0.000 \\
\hline
\end{tabular}

I-frame is the largest due to low bit rate and high distortion of losses.

In Tables 3 and 4, the protection rates of equal error protection (EEP) and Protect-L cases are also given. These protection rates are nonoptimal and will be compared with the proposed optimal protection rates by simulations. In order to construct the EEP case, the resulting bit rate of proposed protection is distributed to the layers so that each layer has the same protection ratio. Protect-L case is constructed similarly, using the results of [31], where the bit rate of protection is distributed to only layers of left view (layer 1 and layer 0 ) so that these layers have the same protection ratio. The encoder bit rates for EEP and Protect-L are the same as the optimal streaming case.

7.2. Simulation Results. In this section, we evaluate the performance of the proposed stereoscopic video streaming system on lossy channels via simulations. We use two stereoscopic videos "Rena" (Camera 38, 39) $(640 \times 480$, first 30 frames $)$ and "Soccer" $(720 \times 480$, first 30 frames $)$ for performance evaluation. We encode the stereoscopic videos with the bit rates obtained by the minimization in (21) for given $p_{e}$ and $R_{C}$, and NAL unit size is fixed to 150 bytes. The number of NAL units per layer can be calculated by dividing the given encoder bit rate to NAL unit size which yields the number of input symbols for the channel coder.

For channel protection, we use systematic Raptor codes based on their suitability for our case as explained in
Section 4. We applied Raptor encoding to the source encoded video data using the protection rates obtained by the minimization in (21) for given $p_{e}$ and $R_{C}$. The proposed optimal streaming scheme is compared with EEP, Protect-L, no-loss, and no-protection cases. The no-loss case represents the quality of the video when the stereoscopic video is encoded with all available channel bandwidth and no transmission occurs. The no-protection case represents the transmission of the video of no-loss case without any channel protection and only error concealment is used at the decoder.

The simulation results give the average of 100 independent lossy transmission simulations for each $p_{e}$ and $R_{C}$, where each packet is lost with a probability of $p_{e}$. Simulation results are based on the weighted PSNR measure. If we denote the average left and right per pixel distortions in MSE as $D_{\text {left }}$ and $D_{\text {right }}$, then the total PSNR distortion $D(\mathrm{~dB})$ can be calculated as

$$
D(\mathrm{~dB})=10 \cdot \log _{10}\left(\frac{255^{2}}{(2 / 3) D_{\text {left }}+(1 / 3) D_{\text {right }}}\right) .
$$

We give the simulation results of stereoscopic video pair "Rena" in Figures 18 to 21 and those of "Soccer" in Figures 22 to 25. The gap between the results of the no-loss and the proposed case is caused by the reduction of the encoder bit rates of video where the remaining bit rate is used for channel protection. The simulation 


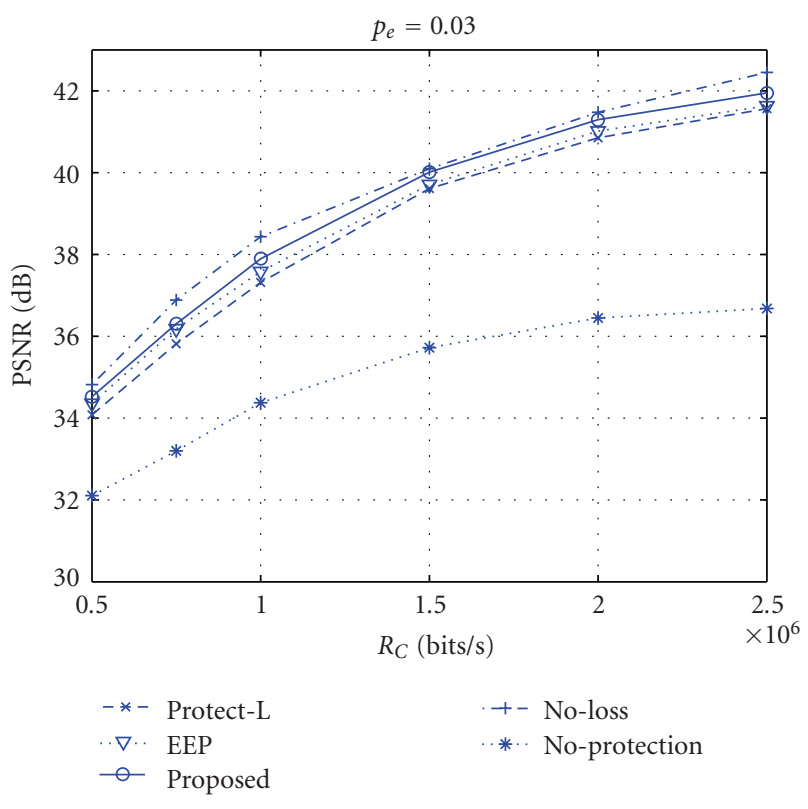

FIGURe 18: Results for $p_{e}=0.03$ for "Rena" video.

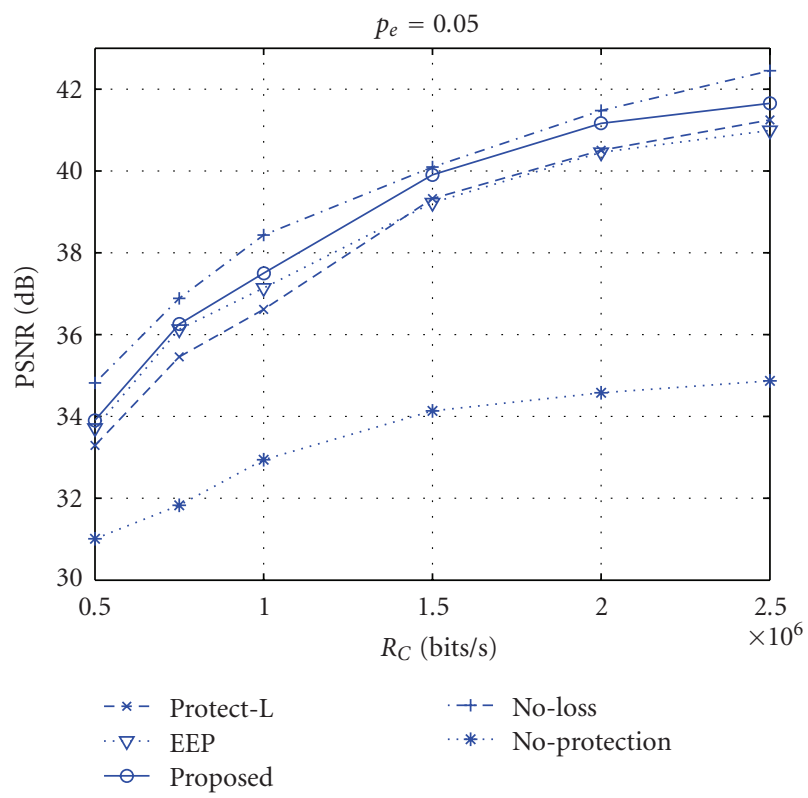

Figure 19: Results for $p_{e}=0.05$ for "Rena" video.

results demonstrate the superiority of the proposed scheme compared to nonoptimized schemes. For low bit rates, the difference is not clear but for high bit rates the difference is $1 \mathrm{~dB}$ for $p_{e}=0.10$ and nearly $2 \mathrm{~dB}$ for $p_{e}=0.20$. The results of the no-protection case clearly point out the need for FEC utilization in stereoscopic video streaming.

\section{Conclusions}

In this work, we presented a rate-distortion optimized error-resilient stereoscopic video streaming system with Raptor codes and evaluated its performance via simulations.

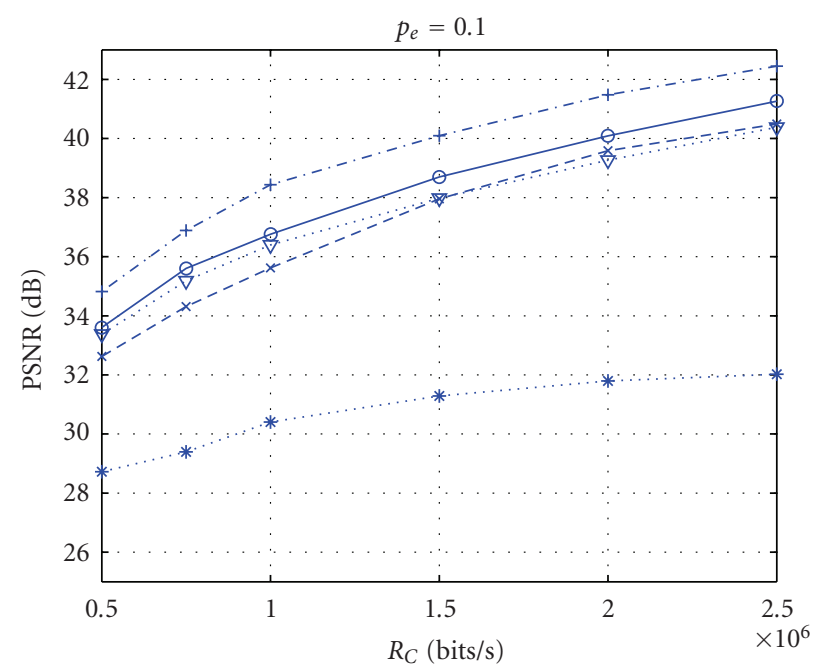

$\begin{array}{ll}-*-\text { Protect-L } & +- \text { No-loss } \\ \because \nabla \cdot \cdot \text { EEP } & * \cdot \cdot \text { No-protection } \\ - & - \text { Proposed }\end{array}$

Figure 20: Results for $p_{e}=0.10$ for "Rena" video.

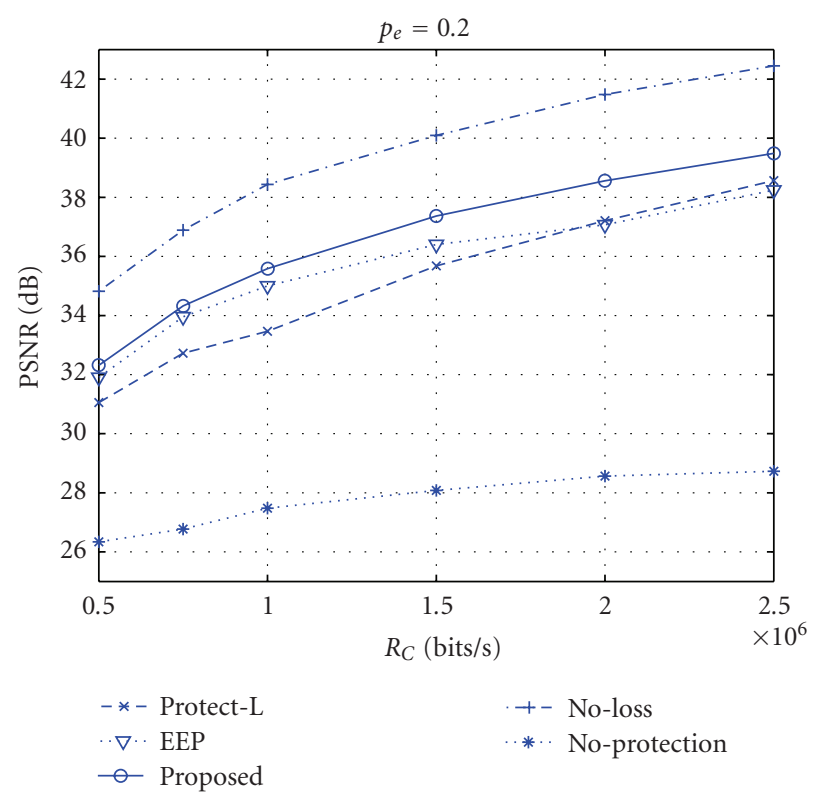

FIgURE 21: Results for $p_{e}=0.20$ for "Rena" video.

We investigated all aspects of an end-to-end stereoscopic streaming system. Initially, we defined the layers of the stereoscopic video which have interdependencies. Then, we obtained the analytical models for the RD curve of these layers where we extended the model of monoscopic video for the dependent layers of stereoscopic video. We showed that the analytical model of the $\mathrm{RD}$ curve accurately approximates the actual RD curve of the layers. Then, we obtained the analytical model of Raptor codes, which also accurately approximates the actual performance. Then, we estimated the transmission distortion for each layer where we also considered the propagation of NAL unit losses to following 


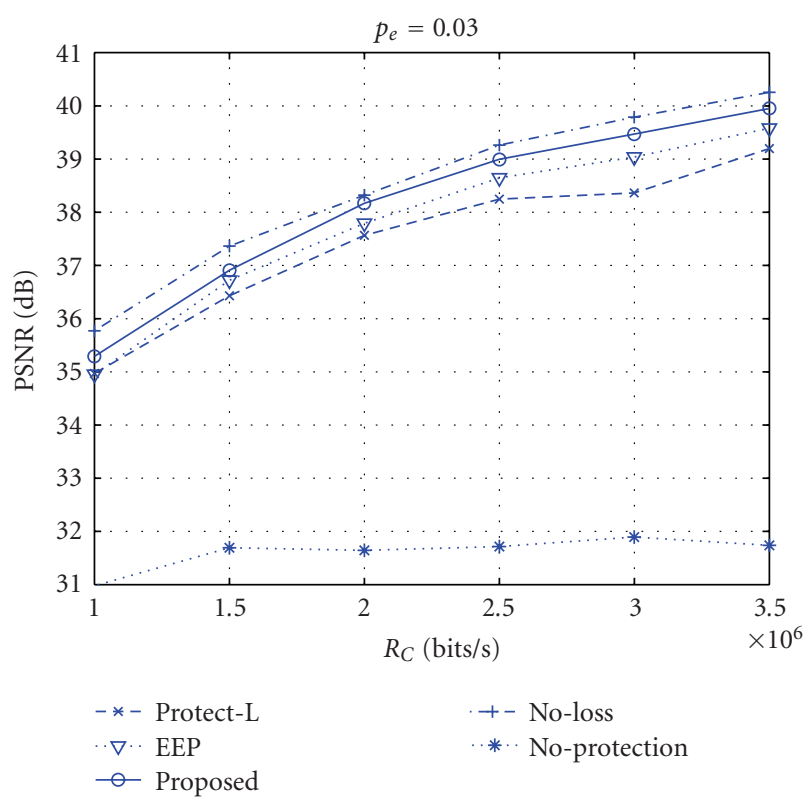

Figure 22: Results for $p_{e}=0.03$ for "Soccer" video.

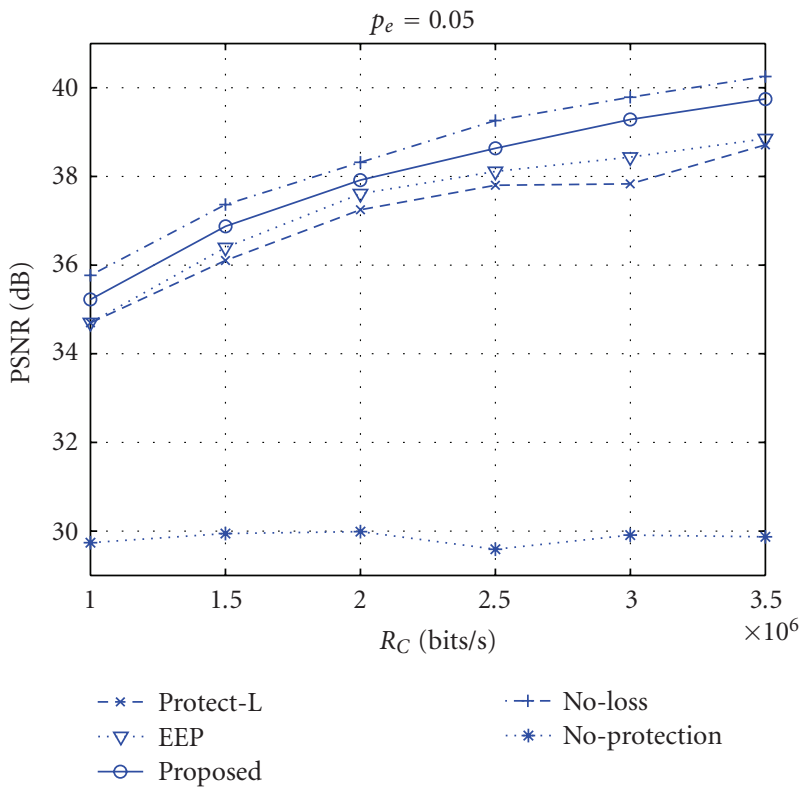

FIgure 23: Results for $p_{e}=0.05$ for "Soccer" video.

frames. Finally, we combined the two analytical models and the estimated transmission distortions in an end-to-end distortion minimization to obtain optimal encoder bit rates and UEP rates for the defined layers.

We evaluated the performance of the system via simulations where we used two stereoscopic videos "Rena" and "Soccer," which have different video characteristics. For both of the videos, the simulation results yielded the superiority of the proposed system compared to nonoptimized schemes. Also, the necessity of the utilization of FEC codes, such as Raptor codes, for stereoscopic video streaming on lossy transmission channels is clearly observed by examining the quality gap between the protected and nonprotected streaming schemes.

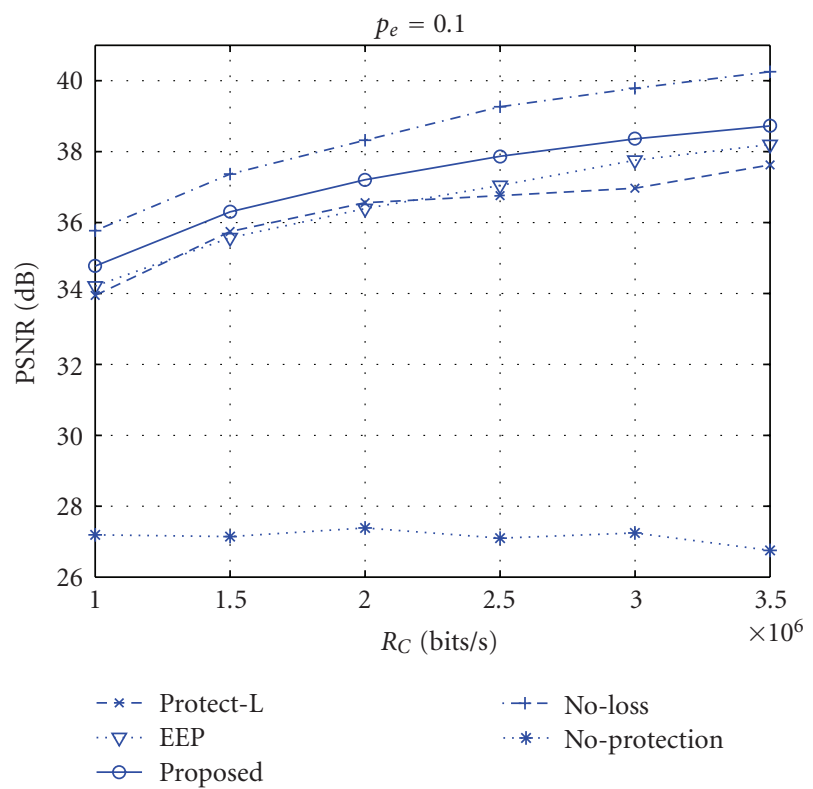

FIGURE 24: Results for $p_{e}=0.10$ for "Soccer" video.

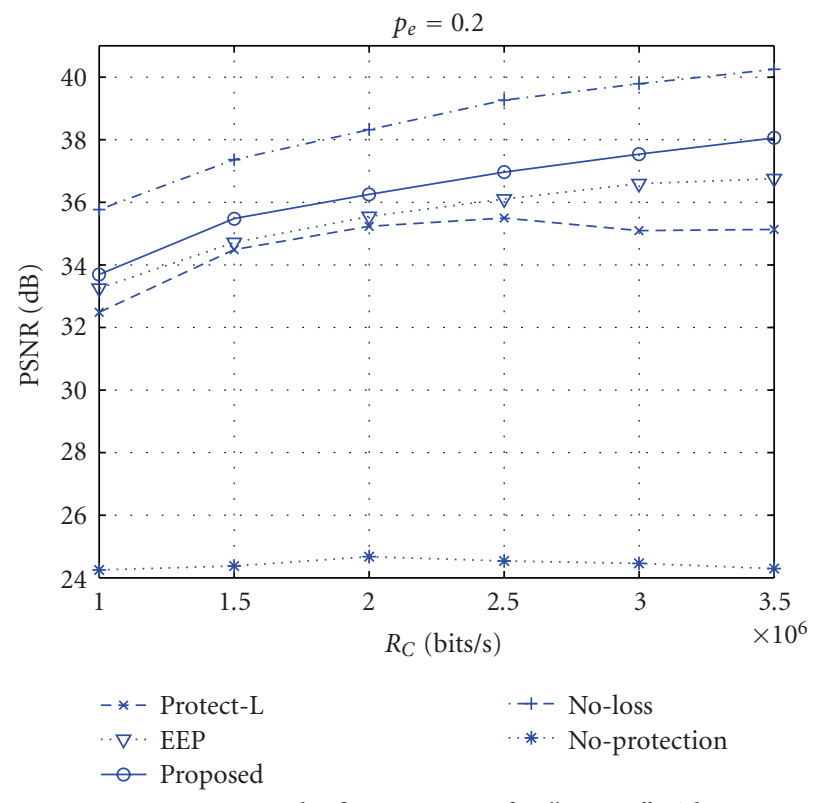

FIGURE 25: Results for $p_{e}=0.20$ for "Soccer" video.

The proposed system can be applied to any layered stereoscopic or multiview streaming system for error resiliency. Future research can evaluate the performance of the proposed system for multiview video streaming, where achieving superior results can be predicted by examining the results of this work.

\section{Acknowledgments}

This work was supported by the EC under Contract FP6511568 3DTV and in part by TÜBİTAK (Scientific and Technical Research Council of Turkey) under Contract BTTTurkiye 105E065. The first and second authors are supported in part by TÜBİTAK. 


\section{References}

[1] L.-J. Lin and A. Ortega, "Bit-rate control using piecewise approximated rate-distortion characteristics," IEEE Transactions on Circuits and Systems for Video Technology, vol. 8, no. 4, pp. 446-459, 1998.

[2] J. I. Ronda, M. Eckert, F. Jaureguizar, and N. Garcia, "Rate control and bit allocation for MPEG-4," IEEE Transactions on Circuits and Systems for Video Technology, vol. 9, no. 8, pp. 1243-1258, 1999.

[3] J. Ribas-Corbera and S. Lei, "Rate control in DCT video coding for low-delay communications," IEEE Transactions on Circuits and Systems for Video Technology, vol. 9, no. 1, pp. 172-185, 1999.

[4] Y. Sermadevi and S. S. Hemami, "Linear programming optimization for video coding under multiple constraints," in Proceedings of the Data Compression Conference (DCC '03), pp. 53-62, Snowbird, Utah, USA, March 2003.

[5] J. Chakareski, J. Apostolopoulos, and B. Girod, "Lowcomplexity rate-distortion optimized video streaming," in Proceedings of the International Conference on Image Processing (ICIP '04), vol. 3, pp. 2055-2058, Singapore, October 2004.

[6] E.-H. Yang and X. Yu, "Rate distortion optimization for H.264 interframe coding: a general framework and algorithms," IEEE Transactions on Image Processing, vol. 16, no. 7, pp. 1774-1784, 2007.

[7] P. A. Chou and Z. Miao, "Rate-distortion optimized streaming of packetized media," IEEE Transactions on Multimedia, vol. 8, no. 2, pp. 390-404, 2006.

[8] E. Setton and B. Girod, "Rate-distortion analysis and streaming of SP and SI frames," IEEE Transactions on Circuits and Systems for Video Technology, vol. 16, no. 6, pp. 733-743, 2006.

[9] G. J. Conklin, G. S. Greenbaum, K. O. Lillevold, A. F. Lippman, and Y. A. Reznik, "Video coding for streaming media delivery on the Internet," IEEE Transactions on Circuits and Systems for Video Technology, vol. 11, no. 3, pp. 269-281, 2001.

[10] B. Girod, K. Stuhlmueller, M. Link, and U. Horn, "Packet-lossresilient Internet video streaming," in Visual Communications and Image Processing, vol. 3653 of Proceedings of SPIE, pp. 833 844, San Jose, Calif, USA, January 1999.

[11] H. Cai, B. Zeng, G. Shen, Z. Xiong, and S. Li, "Error-resilient unequal error protection of fine granularity scalable video bitstreams," EURASIP Journal on Applied Signal Processing, vol. 2006, Article ID 45412, 11 pages, 2006.

[12] Y. Pei and J. W. Modestino, "H.263+ packet video over wireless IP networks using rate-compatible punctured turbo (RCPT) codes with joint source-channel coding," in Proceedings of the International Conference on Image Processing (ICIP '02), vol. 1, pp. 541-544, Rochester, NY, USA, September 2002.

[13] J. W. Byers, M. Luby, M. Mitzenmacher, and A. Rege, "A digital fountain approach to reliable distribution of bulk data," Computer Communication Review, vol. 28, no. 4, pp. 56-67, 1998.

[14] M. Luby, "LT codes," in Proceedings of the 43rd Annual IEEE Symposium on Foundations of Computer Science (FOCS '02), pp. 271-280, Vancouver, Canada, November 2002.

[15] P. Maymounkov, “Online codes," Tech. Rep. TR2002-833, New York University, New York, NY, USA, November 2002.

[16] A. Shokrollahi, "Raptor codes," IEEE Tansactions on Information Theory, vol. 52, no. 6, pp. 2551-2567, 2006.

[17] J.-P. Wagner, J. Chakareski, and P. Frossard, "Streaming of scalable video from multiple servers using rateless codes," in Proceedings of the IEEE International Conference on Multimedia and Expo (ICME '06), pp. 1501-1504, Toronto, Canada, July 2006.
[18] M. Luby, T. Gasiba, T. Stockhammer, and M. Watson, "Reliable multimedia download delivery in cellular broadcast networks," IEEE Transactions on Broadcasting, vol. 53, no. 1, part 2, pp. 235-245, 2007.

[19] M. Luby, M. Watson, T. Gasiba, T. Stockhammer, and W. $\mathrm{Xu}$, "Raptor codes for reliable download delivery in wireless broadcast systems," in Proceedings of the 3rd IEEE Consumer Communications and Networking Conference (CCNC '06), vol. 1, pp. 192-197, Las Vegas, Nev, USA, January 2006.

[20] P. Y. Yip, J. A. Malcolm, W. A. C. Fernando, K. K. Loo, and H. K. Arachchi, "Joint source and channel coding for H.264 compliant stereoscopic video transmission," in Proceedings of the Canadian Conference on Electrical and Computer Engineering (CCECE '05), pp. 188-191, Saskatoon, Canada, May 2005.

[21] A. Vetro, A. Pandit, H. Kimata, and A. Smolic, "Joint draft 4.0 on multiview video coding," JVT-X209, Geneva, Switzerland, June-July 2007.

[22] C. Bilen, A. Aksay, and G. B. Akar, "A multi-view video codec based on H.264," in Proceedings of the IEEE International Conference on Image Processing (ICIP '06), pp. 541-544, Atlanta, Ga, USA, October 2006.

[23] V. Varsa, M. M. Hannuksela, and Y. Wang, "Non-normative error concealment algorithms," ITU-T VCEG-N62, September 2001.

[24] A. Aksay, C. Bilen, E. Kurutepe, et al., "Temporal and spatial scaling for stereoscopic video compression," in Proceedings of the 14th IEEE European Signal Processing Conference (EUSIPCO '06), Florence, Italy, September 2006.

[25] K. Stuhlmüller, N. Färber, M. Link, and B. Girod, "Analysis of video transmission over lossy channels," IEEE Journal on Selected Areas in Communications, vol. 18, no. 6, pp. 1012-1032, 2000.

[26] J. J. Moré, "The Levenberg-Marquardt algorithm: implementation and theory," in Numerical Analysis, vol. 630 of Lecture Notes in Mathematics, pp. 105-116, Springer, Berlin, Germany, 1977.

[27] R. G. Gallager, L.D.P.C. Codes, MIT Press Monograph, Cambridge, Mass, USA, 1963.

[28] M. Luby, A. Shokrollahi, M. Watson, and T. Stockhammer, "Raptor forward error correction scheme for object delivery," RFC 5053, June 2007, http://www.ietf.org/rfc/rfc5053.txt.

[29] N. Ozbek, A. M. Tekalp, and E. T. Tunali, "Rate allocation between views in scalable stereo video coding using an objective stereo video quality measure," in Proceedings of the IEEE International Conference on Acoustics, Speech and Signal Processing (ICASSP '07), vol. 1, pp. 1045-1048, Honolulu, Hawaii, USA, April 2007.

[30] P. E. Gill, W. Murray, and M. H. Wright, Practical Optimization, Academic Press, London, UK, 1981.

[31] A. S. Tan, A. Aksay, C. Bilen, G. B. Akar, and E. Arikan, "Error resilient layered stereoscopic video streaming," in Proceedings of the International Conference on True Vision Capture, Transmission and Display of 3D Video (3DTV '07), Kos Island, Greece, May 2007. 